Covered in: Web of Sciences (WOS); EBSCO; ERIH+; Google Scholar; Index Copernicus; Ideas RePeC; Econpapers; Socionet; CEEOL; Ulrich ProQuest; Cabell, Journalseek; Scipio; Philpapers; SHERPA/RoMEO repositories; KVK; WorldCat; CrossRef; CrossCheck

2018, Volume 10, Issue 1, pages: 244-256| doi: https://doi.org/10.18662/rrem/31

\section{The Effect of}

Cognitive Inhibition

Training on the

Reduction of

Attention-deficit/

Hyperactivity

Symptoms and the

Improvement of

Planning

Performance

\section{Peyman KARIMI}

GOODARZI ${ }^{1}$,

Mohsen RAFIKHAH $*^{2}$,

Amir MOHSEN

RAHNEJAT ${ }^{3}$,

Mohaddese

\section{ESKANDARIPOOR ${ }^{4}$}

${ }^{1}$ Department of Neurosurgery,

AJA University of Medical

Sciences, Tehran, Iran

${ }^{2} \mathrm{Ph}$. Candidate in Psychology,

University of Tehran, Researcher of

AJA University of Medical Sciences,

Tehran, Iran

${ }^{3}$ Department of Psychology, AJA

University of Medical Sciences,

Tehran, Iran

${ }^{4} \mathrm{MA}$ in Educational Psychology,

Semnan University, Semnan, Iran

\begin{abstract}
Background and aims: The deficit in inhibition has been recognized as a central deficit in ADHD. the aim of this study was to investigate the effect of cognitive inhibition training on the reduction of attention-deficit and hyperactivity/impulsivity symptoms and the improvement of planning performance.

Methods: Using cluster sampling, 42 children (8 to 11 years old) of the Iranian army personnel were selected from 3 boys' primarily schools. The diagnosis of students with ADHD was done using Conner's Teachers Rating Scale and the clinical psychologist's assessment. The participants were randomly placed into two groups: experimental group $(n=27)$ and control group $(n=15)$. All participants were assessed in terms of IQ before the intervention. Both of the groups were assessed by The Child Symptom Inventory-4 and Tower of London test before and after the intervention.

Results: The results indicated that cognitive inhibition training resulted in the reduced attention-deficit symptoms and the improved planning performance $(\mathrm{P}<0.05)$; however, there was not a significant difference between the two groups in terms of Hyperactivity/Impulsivity.

Conclusion: This study imply that cognitive inhibition training is effective in reducing attention deficit symptoms and improving planning performance to a great extent.
\end{abstract}

Keywords: Cognitive inhibition, ADHD, Planning, Executive functions.

How to cite: Karimi Goodarzi, P., Rafikhah, M., Mohsen Rahnejat, A., \& Eskandaripoor, M. (2018). The Effect of Cognitive Inhibition Training on the Reduction of Attention-deficit/ Hyperactivity Symptoms and the Improvement of Planning Performance. Revista Romaneasca pentru Educatie Multidimensionala, 10(1), 244-256. https://doi.org/10.18662/rrem/31 


\section{Introduction}

In the fifth edition of Diagnostic and Statistical Manual of Mental Disorders (DSM-5), Attention-Deficit/Hyperactivity Disorder (ADHD) is characterized by persistent inattention and/or hyperactivity/impulsivity that interfere with function or development (Widiger \& Douglas, 2013). ADHD can be caused by a number of factors; including genetic, environmental, neurobiological, and neuropsychological factors (2-(Sonuga-Barke, 2005) The presentation of the Barkley's Theory of ADHD in the recent years has increased interest in the study of inhibitory control in children with ADHD (R. A. Barkley, 1997). According to this theory, ADHD is related to the executive functions (EFs) deficits in the frontal lobe and particularly to the inhibition. Various recent studies have proved this theory (Quay, 1997), (S. J. Johnstone, Roodenrys, Phillips, Watt, \& Mantz, 2010). The executive function is an umbrella term that encompasses the set of higher-order processes such as planning, working memory, inhibition, shifting, and impulse control (R. A. Barkley, 1997), (Hill, 2004), (Miyake et al., 2000). In addition to having a common place in the prefrontal cortex, these EF components interact with each other. For example, Barkley in a study on the children with ADHD, remarked that some cognitive components like the working memory are dependent on inhibition so as to properly function (R. A. Barkley, 1997). Gilhooly believed that many problem-solving and planning processes need a proper processing of the working memory (Gilhooly, 2005). The working memory also plays an important role in the shifting function. Moreover, Asato etal concluded that planning is influenced by the development of inhibition and the working memory and is directed by them (Asato, Sweeney, \& Luna, 2006). Furthermore, the inhibition may be effective in the information retrieval process from the working memory such that it suppresses the unrelated information and facilitates the information retrieval process (Alexander et al., 2002). Cain points out that inhibitory processing operates as a regulator system for the working memory which can prevent the unrelated information from entering the memory (Cain, 2006).

Most studies have emphasized the Inhibition and working memory are the basis of the other EFs. However, it is a question whether the inhibition is the cause of the individual differences in the working memory capacity or its consequence. Although some evidence indicates the effectiveness of the working memory on the inhibition performance (Gorfein \& MacLeod, 2007), Clair-Thompson reported that the working 
memory does not affect inhibition. He studied 38 children with a poor working memory, and 38 age-matched controls. His findings revealed that children with a poor working memory achieved significantly lower scores on measures of planning and attention, but not shifting and inhibition (St ClairThompson 2011). Other studies also indicated that inhibition can influence the working memory function. Cain believes that the individuals with poor inhibition performance will be deficient in their working memory function (Cain, 2006). Moreover, it is reported that the students with a better inhibition performance will do better in the information retrieval process from the working memory. Investigating the relationship between these components, it seems that inhibition plays a key role in managing the working memory and the other EFs.

From etiology perspective, the deficit in inhibition may explain factors such as distraction, neglect, and impulsivity in children with ADHD. The inhibition deficits in these children result in the emergence of impulsive behaviors such that before he understands the task, the child processes the information related to which and answers it. Furthermore, the child's attention is easily deviated by disturbing stimuli and he fails to correct the inappropriate answers. The large role that Inhibitory control seems to play in other EFs has led researchers to develop interventions aimed at improving inhibition. Moreover, the brain imaging of the individuals under cognitive inhibition training indicated considerable changes in their brain cortex function (Houde et al., 2000). Barkley considered the inhibition as an important element for self-discipline and controlling the behaviors of the children with ADHD (R. A. Barkley, 1997).

It should be noted that Barkley stressed the response or behavioral inhibition. Response inhibition involves three interrelated processes: (a) inhibition of the initial pre-potent response to an event, (b) stopping of an ongoing response pattern and (c) protecting this period of delay from disruption by competing events ("interference control") (R. A. Barkley, 2014); However, one of the sustainable deficits emerging in ADHD is the deficits in perception problems, information processing, and the working memory (R. A. Barkley, 1997), (Nigg \& Casey, 2005); it is directly related to cognitive processes. Therefore, this study separates the behavioral inhibition from cognitive inhibition which is the ability to keep active the necessary information and suppress the irrelevant and unnecessary information (Miyake et al., 2000); it seeks to examine the hypothesis of the effectiveness of the cognitive inhibition intervention on the children with ADHD. Regarding the deficits related to inhibition in the children with ADHD and the effect of inhibition on the other EF components, this study was 
conducted and aimed at investigating the effect of cognitive inhibition training on the reduction of attention-deficit (AD) and hyperactivity/impulsivity (HI) symptoms and the improvement of planning performance in the students with ADHD.

\section{Method}

The Medical Research Ethics Committee of the AJA University of Medical Sciences approved this study. Informed consent was obtained of the parents before testing. Children were tested individually one week prior to the start and one week after the last session.

\section{Participants}

This study was conducted on the children of The Islamic Republic of Iran Army personnel who had received an ADHD diagnosis. The sample included 42 students aged between 8 and 11 (age average, $8.9 \pm 0.94$ ). all students were from 3 elementary boys' schools in Tehran, the capital of Iran. The sampling method was in a way that, in the first stage, the teachers of each of the grades were asked to complete Conner's Teachers Rating Scale with regard to each of the students suspected of having ADHD. More than 100 students were recognized as having ADHD by the teachers; however, since the teachers usually overestimate the recognition of this disorder, the parents were interviewed and the psychologist assessed the students so as to identify the true cases. Overall, 42 students were selected to participate in the study and randomly divided into experimental $(\mathrm{n}=27)$ and control $(n=15)$ groups. All participants were assessed in terms of IQ before the intervention. The demographic information and the comparison of the groups in terms of the variables of age and IQ are presented in Table 1.

The inclusion criteria included: (1) receiving a ADHD diagnosis; (2) having an IQ $>70$ (3) the parents' written consent; (4) the ability to read the words; (5) having a working parent in The Islamic Republic of Iran Army; (6) not using ADHD medications; (7) lack of any comorbid disorder based on the school psychologist's diagnosis.

\section{Instruments}

\section{Raven's Colored Progressive Matrices (RCPM)}

RCPM is a non-verbal measure of fluid intelligence in which the participant is shown colored illustrations with one part missing. The participant is asked to identify and select the missing element that completes the pattern from six possible choices. The studies indicate that RCPM is of a desirable validity and reliability in the Iranian children. 


\section{Conner's Teachers Rating Scale (CTRS)}

The translated Persian version of CTRS was used in the present study. CTRS is a commonly used measure of behavioral problems associated with ADHD. The Conner's Teachers Rating Scale was used for screening the ADHD cases in the present study. The Cronbach's alpha of this scale was 0.87 .

\section{The Child Symptom Inventory-4 (CSI-4): Parent Checklist}

CSI-4 is a screening test for behavioral symptoms of many DSM-IV children disorders including ADHD. The ADHD symptoms contained 18 items including nine for ADHD-AD, and nine for ADHD-HI; all of the items are used for ADHD-CT. The parent checklist was used in the present study. Different methods have been considered for the cut-point of this scale, the best of which is 6 . This cut-point has been used in the present study.

\section{Tower of London (ToL)}

The ToL was used to evaluate planning performance. This test includes 3 vertical pegs of different lengths and 3 colored beads in blue, green, and red colors. individuals must move beads from a prearranged sequence on three different pegs to match a goal state determined by the examiner. This test includes 12 stages and the maximum score a participant can obtain as the total planning score will be 36. Different scores are recorded in the ToL; total score and planning time are used as the planning performance index in this study.

\section{Training program}

Using correlation matrix and doing factor analysis among 6 assignments related to cognitive inhibition, Wang etal achieved 3 categories of cognitive inhibition, including graph inhibition, word inhibition, and number inhibition (Wang, Tasi, \& Yang, 2012). This study also uses this general categorization such that the similar tasks were separated based on each of the aforementioned categories and the training related to cognitive inhibition were provided. Using the general structure of the assignments related to cognitive inhibition and applying some modifications in their execution method, the training programs of this study were designed in a way that they can be performed for the Persian-speaking students of the elementary school. The training program is described as follows:

1. Graphic inhibition category

- Some pictures of chimeric animals were shown to the students; the head and body of the animals were differently joined together. The 
participants were asked to, according to the presented instruction, say the name of the animal sometimes based on its head and sometimes based on its body. This task was presented in four stages, the first of which aimed at getting familiar with the original animal shape. Stages two and three included the main exercises related to cognitive inhibition. In the last stage, the participants were asked to push a button on the keyboard if the head and body of an animal matched.

- Different geometric figures were presented (square, triangle, circle, and rhombus) with black ink; there were different smaller figures in light gray color in varying positions of the larger objects. Students were instructed to name the smaller object.

- This task is greatly similar to the previous one. The difference was such that instead of using smaller geometric figures in varying positions of the main object, both of the shapes had nearly the same size and a shape was located in the center of the other shape. The task was done in four stages. In the first and third stages, each participant was asked to inhibit the outer shape and tell the name of the inner shape. This was done in reverse in the second and fourth stages.

- The geometric figures consisting of smaller shapes were shown in this task. There were two stages: 1) the inhibition of the main object and mentioning its constituent figures 2) the inhibition of the constituent figures and mentioning the main object.

- Although the last task of this category was of a simple structure, it demanded much effort for inhibition. Two figures (square and circle) were simultaneously and repeatedly presented on the screen. They were different in terms of size and color. The task consisted of two stages as it is explained. In the first stage, if the size of the square was larger than the circle, the participant should say square and if the circle was larger than the square, he should refer to its color. For example, if the red circle was larger than the blue square, the correct answer was "red", not "circle". This was changed in the second stage. If the square was larger than the circle, the color of which should be mentioned and if the circle was larger than the square, the figures name should be mentioned.

2. Word inhibition category

- The participant was given some pictures of animals and there was a difference between the picture of the animal and the word placed on which. In this task students were required to say the name of the animal and inhibit the word.

- A big letter of the Persian alphabet was presented each time in this exercise that the letter itself was composed of smaller letters. (For example, a 
big $\mathrm{S}$ composed of small Ts). The two stages of this exercise included: 1) mentioning the large letter and inhibiting the small ones; 2) mentioning the small letters and inhibiting the large one.

- The word-color inhibition: generally, the structure of this task is to present the name of each color with a different color. This task was done in two stages. In the first stage, the student needed to quickly read a set of words, each of which was the name of a color. In the second stage, participants were required to name the color of the ink instead of reading the word. For example, if the word "GREEN" was printed in a red color, the correct answer was "RED"

3. Number inhibition category

- This task consisted of a series of digits and each Student should read them as fast as possible. The next stage, he had to name the number of stimuli within the series. For example, if series "444" was presented, the correct answer was " 3 ".

- The even and odd numbers were randomly presented on the screen and, then, a figure (ship) was quickly shown in green and red colors. The participants were asked to quickly push a button on the keyboard only if the even number was shown after a green ship.

- The pairs of single digits (ranging from 1 to 9) were presented. One of the digits was printed in a large font and one in a smaller font. Each participant must determine which number has the higher value while ignoring their sizes.

All tasks and trains were presented on a 14" screen. The available pictures in the tasks were displayed in 1-second intervals; hence, the participants needed to quickly answer as much as possible. After doing every task, the participants were instructed how to only pay attention to the main stimulus by inhibiting the disturbing stimuli. The intervention was done individually in a room with complete silence. The training program lasted 10 sessions, and each daily session lasted $30 \mathrm{~min}$.

\section{Results}

SPSS 21 software was used for data analysis. First, KolmogorovSmirnov test was used to check the data distribution. Results showed that all data were normally distributed. We used a Multivariate Analysis of covariance (MANCOVA) to analyze differences between the experimental and control groups. Table 1 presents distribution of frequency of $\mathrm{AD}, \mathrm{HI}$, and combination of these in study groups. 
Table 1. Participant characteristic

\begin{tabular}{rrrrr}
\hline & $\begin{array}{r}\text { Experimental group } \\
\mathrm{N}=27\end{array}$ & $\begin{array}{r}\text { Control group } \\
\mathrm{N}=15\end{array}$ & $\mathrm{t}$ & $\mathrm{p}$ \\
& Mean (SD) & Mean (SD) & & \\
\hline IQ & $99.56(9.37)$ & $97.53(9.41)$ & 1.37 & .18 \\
Age (years) & $8.6(.8)$ & $9.2(1.08)$ & .67 & .51 \\
ADHD subtypes & & & & \\
AD & $14(51.9 \%)$ & $8(53.3 \%)$ & & \\
Combined & $9(33.3 \%)$ & $6(40 \%)$ & & \\
\hline
\end{tabular}

Note: $\mathrm{AD}=$ Attention Deficit; $\mathrm{HI}=$ Hyperactive/Impulsivity

This table also shows the participants' demographic characteristics. A t-test was used to compare the means of IQ and age. As it is shown, there was no statistically significant difference in the IQ and age of the two groups.

\section{The effect of cognitive inhibition training on reducing $A D / H D$ symptoms}

There was a significant difference between the two experimental and control groups in AD; such that the experimental group did better in the posttest. $\mathrm{F}(1,38)=6.33, \mathrm{p}=0.02, \mathrm{ES}=15$ (Table 2$)$. The calculated effect size for this variable is 0.15 ; this indicates that $15 \%$ of the changes in this variable results from cognitive inhibition training.

Table 2. Differences between the pretest and posttest results regarding ToL and SCI-4 for the experimental and control groups

\begin{tabular}{|c|c|c|c|c|c|c|c|}
\hline & \multicolumn{2}{|c|}{$\begin{array}{l}\text { Experimental group } \\
\text { Mean (SD) }\end{array}$} & \multicolumn{2}{|c|}{$\begin{array}{l}\text { Control group } \\
\text { Mean (SD) }\end{array}$} & \multicolumn{3}{|c|}{ MANCOVA } \\
\hline & $\overline{\text { pre }}$ & Post & Pre & Post & $\mathrm{F}$ & $\mathrm{p}$ & $\mathrm{ES}$ \\
\hline $\begin{array}{l}\text { ToL } \\
\text { total score }\end{array}$ & $20.52(3.46)$ & $24.07(3.71)$ & $19.93(5.34)$ & $21.13(4.24)$ & 7.03 & .01 & .16 \\
\hline $\begin{array}{l}\text { Planning } \\
\text { time } \\
\text { SCI-4 }\end{array}$ & $193.26(26.12)$ & $178.37(21.37)$ & $186(27.66)$ & $178.21(18.85)$ & 4.03 & .04 & .10 \\
\hline $\mathrm{AD}$ & $6.22(1.62)$ & $3.48(1.45)$ & $5.80(2.14)$ & $4.53(1.64)$ & 6.33 & .02 & .15 \\
\hline $\mathrm{HI}$ & $5.59(1.80)$ & $4.60(1.72)$ & $4.47(2.32)$ & $4.13(2.29)$ & .33 & .57 & $<.01$ \\
\hline
\end{tabular}

Note: Bold $=\mathrm{p}<.05 ; \mathrm{ES}=$ effect size 
We found no effect of the intervention on HI; F $(1,38)=0.33$, p $=$ 0.57 (Table 2). Therefore, it seems that the applied intervention has not had any effect on reducing HI symptoms in the experimental group.

\section{The effect of cognitive inhibition training on the improvement of the planning performance}

Both total score and planning time showed a significant difference in the posttest. The results of data analysis for the total score and planning time were respectively calculated as $\mathrm{F}(1,38)=7.03, \mathrm{p}=0.01, \mathrm{ES}=0.16$ and $\mathrm{F}$ $(1,38)=0.33, \mathrm{p}=0.04, \mathrm{ES}=10$ (Table 2). Therefore, it can be said that cognitive inhibition training resulted in the improvement of the planning performance in the experimental group. Furthermore, the calculated effect size showed that $16 \%$ of the changes in total score and $10 \%$ of the planning time are resulted from the research intervention.

\section{Discussion}

This study aimed at investigating the possibility of reducing $\mathrm{AD}$ and HI symptoms and also improving the planning performance in students with $\mathrm{AD} / \mathrm{HD}$ through cognitive inhibition intervention.

As the findings indicated, the cognitive inhibition training resulted in the reduction of AD symptoms in the children with ADHD. This finding was in line with the studies referring to the effect of the programs related to cognitive inhibition on the attention in the individuals with ADHD (S. J. Johnstone et al., 2010), (20). It can be said that cognitive inhibition training with its special mechanism helped the students who participated in this study increase their focus and attention level through suppressing the disturbing stimuli.

However, the present intervention did not have any effect on reducing the HI symptoms in the experimental group. The causes of this cognitive issue may be the nature of the intervention program and the kind of inhibition deficits in the participants. Barkley believes that the core deficit in ADHD is problem in response inhibition (R. A. Barkley, 1997). This finding was also confirmed in the study done by Engelhardt etal. They conducted a study on a sample with an age range of 13 to 37 years old and concluded that the individuals with ADHD do not have multiple deficits in inhibition and there is merely an integrated deficit in inhibition which is not related to the cognitive system (Engelhardt, Nigg, Carr, \& Ferreira, 2008). However, one should be cautious about generalizing this finding since some studies have regarded the inhibition deficits in the individuals with ADHD as related to the cognitive control mechanism. For example, Johnstone etal 
showed that using multiple cognitive training programs can be effective in reducing the ADHD symptoms (S. J. Johnstone et al., 2012). Furthermore, Ven etal considered the behavioral inhibition to be related to cognitive control functions and especially the working memory (Ven, Kroesbergen, Boom, \& Leseman, 2013). It seems necessary to do more research and use a precise cognitive method with regard to the sample group and the research instruments so as to achieve a common agreement on the existence of a deficit in the cognitive or behavioral system of the individuals with ADHD. However, it is clear that the cognitive inhibition training in the present study has not been effective in reducing the HI symptoms of the sample group. In addition to the differences in the sample of this study with the other similar studies (in terms of age, gender, sample size, kinds of disorder subgroups, and difference in the applied trainings), it is necessary to mention that the intervention in the present study was cognitive and its effect may not manifest in the behavior in short term. In other words, the ineffectiveness of the cognitive inhibition training in HI symptoms may be due to the nature of the program which is cognitive and not behavioral. Moreover, it may be due to the cross-sectional intervention of the research which demands a long-term follow-up.

As shown in Table 2, the results indicated the effectiveness of the cognitive inhibition training in both scores. In other words, the planning performance in the experimental group was better than the control group which did not receive any interventional program. These findings are in line with the studies confirming the effect of inhibition on planning performance (Asato et al., 2006), (Bull, Espy, \& Senn, 2004). Bull etal came to conclusion that inhibition is the best predictor of the participants' performance in the Tower of London test (Bull et al., 2004). In the present study, it is also possible that the inhibition performance improvement through the applied intervention has resulted in the improvement of planning performance in the children with ADHD.

Despite the effectiveness of the cognitive inhibition intervention in the performance of the Tower of London, it should be born in mind that an inhibition process may not be a predictor of a better performance in planning by itself. Studies have indicated that it is the multiple cognitive processes that influence the performance of the Tower of London test. For example, Bishop etal showed that, instead of only one inhibition process, complicated processes like shifting are involved in doing the tower tasks (Bishop, Aamodt-Leeper, Creswell, McGurk, \& Skuse, 2001). Furthermore, the role of the working memory should be taken into consideration. It is believed that the planning performance is affected by the development of 
the working memory and inhibition(Alexander et al., 2002), (Cain, 2006). D'Antuono etal investigated the effective factors in the mental planning performance and concluded that the two components of visuospatial working memory and fluid Intelligence can be predictors of the individuals' performance in the Tower of London test (D'Antuono et al., 2017). From Gilhooly's perspective, the working memory and particularly the visuospatial working memory is a desirable predictor of the participants' performance in the Tower of London test (Alexander et al., 2002). In addition to what mentioned, it is necessary to be noted that the cognitive inhibition training may affect the development of the working memory by itself (May, Hasher, \& Kane, 1999), (Gorfein \& MacLeod, 2007). Inhibition operates as a regulating system for the working memory which prevents the unrelated information from entering the memory (Gorfein \& MacLeod, 2007). Hence, the ability to inhibit the disturbing information leads to the improvement of the working memory performance, too. Therefore, it can be said that the improvement of the participants' planning performance may be due to the direct intervention of cognitive inhibition and the improvement of the working memory performance. This is more confirmed when it is noted that the training programs used in the present study are of a visuospatial kind. As a result, they might have been effective in the improvement of the visuospatial working memory.

\section{Conclusion}

With regard to what mentioned and the analysis of the research hypotheses, it can be said that although the cognitive inhibition training was not effective in reducing the HI symptoms, it had a significant effect on the other variables of the study. Therefore, making some modifications so as to enhance and correct the shortcomings of this intervention program, one can play an important role in reducing the problems related to ADHD and other disorders which have a similar etiology.

\section{Limitations}

The limitations of this study were the small sample size and being limited to the male participants. Furthermore, it was not possible to follow the results in the future because of the time limitation. It is obvious that using follow-up study shows the possibility of assessing the effectiveness or ineffectiveness of the intervention program in the variables in long-term; it can lead to a more precise generalization of the results. 


\section{Disclosure statement}

No potential conflict of interest was reported by the authors.

\section{References}

Alexander, K., S., W. G., Schaaf, J. M., Edelstein, R. S., Quas, J. A., \& Shaver, P. R. (2002). The role of attachment and cognitive inhibition in children's memory and suggestibility for a stressful event. Journal of Experimental Child Psychology, 83(1), 262-290.

Asato, M. R., Sweeney, J. A., \& Luna, B. (2006). Cognitive processes in the development of TOL performance. Neuropsychologia, 44(12).

Barkley, R. A. (1997). Behavioral inhibition, sustained attention, and executive functions: constructing a unifying theory of ADHD. Psychological Bulletin, 121(1).

Barkley, R. A. (2014). Attention-deficit byperactivity disorder: A handbook for diagnosis and treatment:: Guilford Publications.

Bishop, D. V., Aamodt-Leeper, G., Creswell, C., McGurk, R., \& Skuse, D. H. (2001). Individual differences in cognitive planning on the Tower of Hanoi task: Neuropsychological maturity or measurement error? The Journal of Child Psychology and Psychiatry and Allied Disciplines, 42(4).

Bull, R., Espy, K. A., \& Senn, T. E. (2004). A comparison of performance on the Towers of London and Hanoi in young children. Journal of Child Psychology and Psychiatry, 45(1), 743-754.

Cain, K. (2006). Individual differences in children's memory and reading comprehension: An investigation of semantic and inhibitory deficits. Memory, 14(5), 553-569.

D’Antuono, G., La Torre, F. R., Marin, D., Antonucci, G., Piccardi, L., \& Guariglia, C. (2017). Role of working memory, inhibition, and fluid intelligence in the performance of the Tower of London task. Applied Neuropsychology: Adult, 24(6), 548-580.

Engelhardt, P. E., Nigg, J. T., Carr, L. A., \& Ferreira, F. (2008). Cognitive inhibition and working memory in attention-deficit/hyperactivity disorder. Journal of abnormal psychology, 117(3).

Gilhooly, K. J. (2005). The cognitive psychology of planning. Working memory and planning, 71-88.

Gorfein, D. S., \& MacLeod, C. M. (2007). Inhibition in cognition. Psychological Bulletin.

Hill, E. L. (2004). Executive dysfunction in autism. Trends in cognitive sciences, $8(1), 26-32$. 
The Effect of Cognitive Inhibition Training on the Reduction of Attention-def ... Peyman KARIMI GOODARZI, et. al.

Houde, O., Zago, L., Mellet, E., Moutier, S., Pineau, A., Mazoyer, B., \& Tzourio-Mazoyer, N. (2000). Shifting from the perceptual brain to the logical brain: The neural impact of cognitive inhibition training. . Journal of cognitive neuroscience, 12(5), 721-800.

Johnstone, S. J., Roodenrys, S., Blackman, R., Johnston, E., Loveday, K., Mantz, S., \& Barratt, M. F. (2012). Neurocognitive training for children with and without AD/HD. ADHD Attention Deficit and Hyperactivity Disorders. . ADHD Attention Deficit and Hyperactivity Disorders, 1(4).

Johnstone, S. J., Roodenrys, S., Phillips, E., Watt, A. J., \& Mantz, S. A. (2010). A pilot study of combined working memory and inhibition training for children with AD/HD. ADHD Attention Deficit and Hyperactivity Disorders, 2(1), 31-42.

May, C. P., Hasher, L., \& Kane, M. J. (1999). The role of interference in memory span. . Memory \& cognition, 27(5), 759-767.

Miyake, A., Friedman, N. P., Emerson, M. J., Witzki, A. H., Howerter, A., \& Wager, T. D. (2000). The unity and diversity of executive functions and their contributions to complex "frontal lobe" tasks: A latent variable analysis Cognitive psychology, 1(41), 49-100.

Nigg, J. T., \& Casey, B. J. (2005). An integrative theory of attentiondeficit/hyperactivity disorder based on the cognitive and affective neurosciences. Development and psychopathology, 17(3).

Quay, H. C. (1997). Inhibition and attention deficit hyperactivity disorder. Journal of abnormal child psychology, 25(1), 7-13.

Sonuga-Barke, E. J. (2005). Causal models of attention-deficit/hyperactivity disorder: from common simple deficits to multiple developmental pathways. Biological psychiatry, 57(11).

St Clair-Thompson, H. L. (2011). Executive functions and working memory behaviours in children with a poor working memory. Learning and Individual Differences, 21(4).

Ven, S. H., Kroesbergen, E. H., Boom, J., \& Leseman, P. P. (2013). The structure of executive functions in children: A closer examination of inhibition, shifting, and updating. British Journal of Developmental Psychology, 1(31).

Wang, L. C., Tasi, H. J., \& Yang, H. M. (2012). Cognitive inhibition in students with and without dyslexia and dyscalculia. Research in developmental disabilities, 33(5), 1453-1461.

Widiger, T. A. S., \& Douglas, B. (2013). American Psychiatric Association. Diagnostic and statistical manual of mental disorders: American Psychiatric. 